\title{
I look down on him...
}

DOI:

$10.1038 /$ nrc2237
A classless society would undoubtedly be a good thing, but this might not be true for cancer cells. Recent research published in Cancer Cell by Anthony Letai and colleagues shows that, therapeutically at least, class is important.

Letai and colleagues, and others, have previously shown that the expression and interaction of members of the BCL2 family of cell death proteins (including $\underline{B A X}, \underline{B A K}$ and the $\mathrm{BH} 3$-only proteins) can be

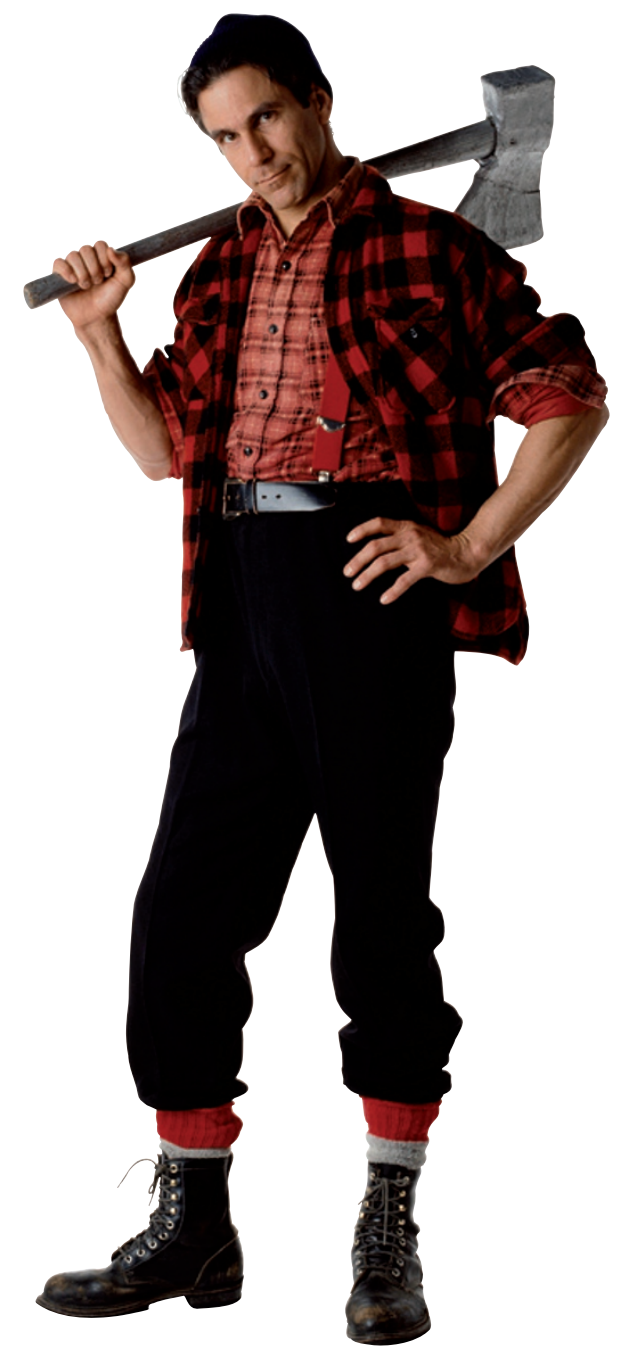

used to identify cells that are primed to undergo apoptosis. Members of the $\mathrm{BH} 3$-only proteins come in two flavours: activators and sensitizers. Activator BH3-only proteins can induce cell death in cells that express BAX and BAK by directly activating them, resulting in the release of cytochrome $c$ from mitochondria owing to mitochondrial membrane permeabilization (MOMP). Sensitizer BH3-only proteins are unable to do this and instead function by displacing activator BH3-only proteins from anti-apoptotic proteins such as BCL2. Cells that are primed for death express anti-apoptotic proteins like BCL2 that are bound to activator $\mathrm{BH} 3$-only proteins like BIM. Exposure to a sensitizer BH3only protein displaces the activator, resulting in BAX and BAK activation, MOMP and apoptosis. Letai and colleagues have now taken this concept further to investigate whether identifying cells that are primed for death has therapeutic significance.

The authors attempted to sort 18 cell lines of diffuse large B cell lymphoma (DLBCL), a disease known for its heterogeneity, into three classes: class A cells in which activation of the $\mathrm{BH} 3$-only cell death proteins is inhibited; class B cells in which the death effector proteins BAX and BAK have reduced expression; and class $\mathrm{C}$ cells that overexpress anti-apoptotic proteins such as BCL2 or MCL1. In line with their previous findings, class $C$ cells should be primed for death because, although they overexpress BCL2 (or other anti-apoptotic members of this family), it is fully bound to activator $\mathrm{BH} 3$-only proteins. So, using a panel of BH3-only peptides and induction of MOMP in mitochondria isolated from the cells as an endpoint, the authors initially separated four DLBCL cell lines into classes. Two were class $\mathrm{C}$ owing to their sensitivity to sensitizer $\mathrm{BH} 3$-only peptides, one was class $\mathrm{A}$, which was sensitive to activator but not sensitizer BH3-only peptides, and one was class $B$, which was insensitive to $\mathrm{BH} 3$-only peptides of either type. These findings were verified through western blot analyses.

Why are these classes important? ABT-737 is a compound currently in clinical trials that is based on a BH3-only domain, and it antagonizes the function of BCL2. The authors showed that their $\mathrm{BH} 3$ profiling screen can identify DLBCL cell lines that are sensitive to ABT-737. Moreover, it is not the levels of BCL2 expression that determine sensitivity to ABT-737, but the levels of the BH3only protein BIM that dictate this. ABT-737 causes the release of BIM and consequent activation of BAX and BAK, leading to apoptosis. Therefore BIM and BCL2 must be expressed for cells to be sensitive to ABT-737. In addition to ABT-737, sensitivity to conventional chemotherapeutic drugs, such as etoposide and vincristine that induce cell death through MOMP can also be predicted by $\mathrm{BH} 3$ profiling; class $C$ cells are more sensitive to these drugs than class A or B cells.

These results show that $\mathrm{BH} 3$ profiling can be used to determine sensitivity to anticancer agents in DLBCL cell lines. Further work is needed to establish whether this extends to cells from solid tumours.

Nicola McCarthy

ORIGINAL RESEARCH PAPERS Deng, J. et al. $\mathrm{BH} 3$ profiling identifies three distinct classes of apoptotic blocks to predict response to ABT-737 and conventional chemotherapeutic drugs. Cancer Cell 12, 171-185 (2007) 